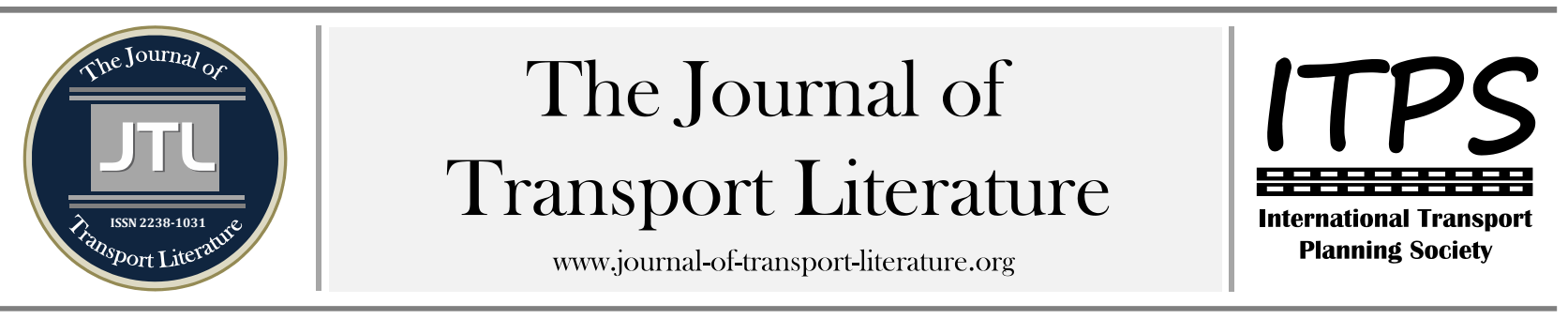

\title{
Instalações humanitárias como alternativa de preparação para eventos sazonais no Estado do Amazonas
}

Thiago Maciel Neto ${ }^{+}$; Mirian Buss Gonçalves

Santa Catarina Federal University, Florianópolis, Brazil

\section{Article Info}

Palavras-chave:

Logística humanitária

Localização de instalações

Planejamento logístico

Submitted 30 Nov 2014

received in revised form 30 Jan 2015

accepted 1 Apr 2015.

Licensed under

Creative Commons

CC-BY 3.0 BR.

\section{Resumo}

As ações na Logística Humanitária podem ser realizadas antes ou após as ocorrências de desastres naturais, destacando-se, nas fases de preparação e resposta, a localização de instalações e a distribuição de suprimentos e transportes de vítimas, respectivamente. No Estado do Amazonas, em decorrência das anormalidades dos fenômenos naturais pré-anunciados, problemas de abastecimento afetam a sobrevivência da população local, principalmente das comunidades interioranas. Para tanto, estratégias logísticas podem ser implantadas para aliviar as necessidades dos amazonenses na ocorrência desses eventos. Deste modo, o objetivo do trabalho é apresentar um modelo conceitual de localização e roteirização para distribuição de suprimentos para instalações humanitárias como alternativa de preparação humanitária para os necessitados em eventos catastróficos sazonais no Estado do Amazonas.

+ Corresponding author. Universidade Federal de Santa Catarina, Departamento de Engenharia de Produção. CEP 88.040-900 - Florianopolis, SC - Brasil.

E-mail address: thiagomn2011@gmail.com.

\section{Introdução}

Este trabalho tem como objetivo apresentar um modelo de localização de instalações humanitárias com proposta de formular uma alternativa na preparação humanitária às pessoas vitimadas por eventos catastróficos sazonais no Estado do Amazonas. Para tanto, é formulado um modelo conceitual de localização de instalações humanitárias, roteirização distribuição de suprimentos usando-se da rede hidrográfica da Bacia Amazônica, aplicado a uma região com eventos sazonais de características singulares no mundo. Também são explicitados os ganhos que podem advir pela adoção desses modelos nas ações de assistência humanitária no Estado do Amazonas.

0 tema investigado partiu de um exame inicial da literatura que indicou as possibilidades de melhorias no atendimento às vítimas de desastres naturais com métodos de localização de instalações humanitárias. A partir destes estudos buscamos identificar qual modelo de localização se adequa melhor à realidade do Estado do Amazonas.

Inicialmente buscaram-se como fonte de pesquisa os problemas logísticos e de acessibilidade nos municípios do Estado do Amazonas pela existência de fenômenos naturais conhecidos na região, problemas cíclicos aos quais a população está habituada e onde já existem ações pontuais que visam mitigar os danos causados; já no caso das secas e cheias, eventos distintos e anômalos na região (provocados por variações pluviométricas causadas por variáveis distintas), é possível visualizar ainda a vulnerabilidade, dependência e carência de ações para minimizar prejuízos e atender de forma rápida e adequada as populações das principais localidades do Estado do Amazonas.

Desta maneira, identificou-se a não existência de uma metodologia que contribuísse na localização de instalações humanitárias no Estado do Amazonas, que permitisse a população um melhor preparo a respeito de questões humanitárias na ocorrência destes eventos sazonais, proporcionando um planejamento de distribuição através do transporte hidroviário na fase de pré-desastre.

Os procedimentos propostos procuram ser compatíveis com os objetivos dos trabalhos encontrados referentes à localização de instalações na logística humanitária, pois visam a preparação das populações atingidas pelos fenômenos mais graves em determinadas regiões que possuem histórico de desastres, para que os objetivos e resultados alcançados pelos autores pesquisados sejam apropriados para tratar os fatores de risco mais representativos no Estado do Amazonas. 0 que se busca é oferecer subsídios para os organismos competentes tomarem ações que contemplem a importância de medidas preventivas adequadas para melhor atender as vítimas destas calamidades no menor tempo possível, considerando a acessibilidade diminuta e os danos que são implícitos nestes processos catastróficos recorrentes no Amazonas.

0 trabalho apresenta uma contribuição científica relevante: à estruturação de um modelo estratégico para o suporte humanitário na região do Amazonas, através da construção de Centros de Abastecimento e Treinamento Humanitários e a roteirização dos suprimentos para atender os principais municípios vitimados por eventos sazonais hidrológicos, evidenciando a importância dos modelos matemáticos na solução de localização de instalações e distribuição de serviços e ajuda humanitária. Essa estratégia apoia-se em uma abordagem conhecida na literatura chamada de Location-Routing Problems (LRPs). Consta-se que esta abordagem é nova para a solução do problema identificado, convencionando-se 
importante, sobretudo tendo em vista a importância que vem sendo atribuído ao tempo de atendimento, custo de transporte e custos operacionais em uma região carente de investimentos nas áreas sociais.

0 artigo é estruturado como segue. Na Seção 1 é apresentado o conceito, referencial teórico relacionado à logística humanitária. Na Seção 2 apresenta-se a situação de seca e enchente no Estado do Amazonas. Na Seção 3 é feita a descrição da proposta de um modelo de alocação de facilidades e distribuição de suprimentos utilizando o modal fluvial como uma alternativa de preparação e atendimento à pessoas na ocorrência de eventos catastróficos sazonais no Estado do Amazonas.

\section{Logística Humanitária}

A partir dos objetivos da logística relacionados à cadeia de abastecimento comercial foi desenvolvido o conceito de logística humanitária que, de forma eficiente e eficaz, vence o tempo e a distância na movimentação de materiais (Nogueira et al., 2008). Diversos estudos voltados à logística humanitária tem recebido destaque no meio acadêmico, destacando-se os desenvolvidos por pesquisadores como Thomas (2004); Balcik et al. (2008); Tomasini e Van Wassenhove (2009); Kovács e Spens (2007); McLachlin e Larson (2011). Segundo Tomasini e Van Wassenhove (2009) é importante que as organizações reconheçam e invistam na logística humanitária em todas as fases, já que após a ocorrência do fato é muito comum requisitar grandes especialistas em logística, perdendo assim fases primordiais do processo. Em suma, é importante amenizar os problemas e suprir as necessidades das comunidades afetadas por desastres. A implantação dos conceitos logísticos adaptados à Logística Humanitária poderá ser um grande diferencial de modo a garantir ações com eficiência e eficácia, permitindo a diminuição do tempo de resposta à situações emergenciais.

\subsection{Cadeia de Suprimentos em Operações Humanitárias}

Os ambientes de ajuda humanitária possuem uma grande variedade de atores, cada um com diferentes conhecimentos, objetivos, interesses, capacidades e logística. Os mecanismos de coordenação referentes à cadeia comercial têm sido bem estudados, ao contrário da coordenação humanitária que ainda está em evolução (OVERSTREET et al. 2011). Kovács e Spens (2007) apontam os atores que compõem a rede de abastecimento de ajuda humanitária (doadores, militares, governos, ONG'S e agências de ajuda) e que os mesmos se distinguem no processo da cadeia. McLachlin e Larson (2011) afirmam que a rápida mudança de prioridades torna a coordenação um desafio na cadeia de suprimentos humanitário, assim como a falta de motivação do lucro, comando e controle.

Dependendo do tipo de desastre e dos atores envolvidos, as características operacionais da cadeia de alívio são diferentes, ou seja, mutáveis de acordo com a situação específica. Nas operações pré-desastre estão incluídas as compras e o pré-posicionamento de estoques, enquanto no pós-desastre o foco principal é a coleta e o transporte (BALCIK et al. 2010)

Thomas e Kopezak (2007) mostram uma classificação especificamente relacionada à estimativa e à distribuição dos recursos, pois na ocorrência de um desastre a assistência humanitária normalmente realiza esse processo padronizado.

A organização de assistência humanitária encaminha uma equipe ao local do desastre para realizar a avaliação das necessidades no prazo de 24 horas. A mesma comunica os resultados de sua avaliação para um responsável fora do local, que faz uma estimativa dos recursos e suprimentos necessários; Aquisição: Dentro de 36 horas do início de um desastre normalmente são realizadas as solicitações de suprimentos ou doações em dinheiro. A procura de suprimentos de fornecedores locais geralmente é a primeira opção dos responsáveis; no entanto, se a organização de assistência humanitária possuir uma central de abastecimento na região, verifica-se a disponibilidade de suprimentos antes, bem como uma lista prévia de fornecedores; Transporte: o transporte dos suprimentos será realizado de forma diferenciada até o local do desastre, dependendo das características do desastre e da região afetada.

Inicialmente os suprimentos são transportados por doadores e pelos fornecedores globais/locais para um centro de distribuição. Esse centro de distribuição é caracteristicamente localizado em grandes centros onde os suprimentos são separados e transferidos para os estoques intermediários/pontos de distribuição. De lá são enviados para os pontos de distribuição local e são entregues para os beneficiários. É importante ressaltar que os fluxos de suprimentos na central de distribuição podem ocorrer também tanto no pré quanto no pós-desastre.

Desta forma, a logística de distribuição de suprimentos passa a ter um papel de fundamental importância para os responsáveis da área humanitária e que devem auxiliar no processo de responsabilidade social levando em consideração os princípios humanitários de humanidade, neutralidade e imparcialidade, para que os mantimentos cheguem para os beneficiários o quanto antes possível.

\subsection{Logística Humanitária e seus desafios}

Nogueira (2010) aponta questões relevantes no momento do desastre ou iminência do mesmo, que devem ser encaradas e respondidas como forma de planejamento de um serviço humanitário. São elas: Onde localizar uma (ou várias) central (ais) de recebimento, controle e distribuição de materiais; Como organizar e controlar o recebimento e a distribuição destes materiais; Como fazer a distribuição na rede. 0 interesse nas localizações das facilidades para logística humanitária está ligado, segundo Doyen et al. (2011), nas estratégias encontradas para o salvamento que devem realçar a eficiência, a distribuição dos suprimentos, a velocidade do transporte e a satisfação da demanda. Problemas similares em abordagem do tipo localização e roteirização são característicos da Logística Humanitária. A modelagem integrada acontece em situações onde a localização de bases e roteirização de veículos são muito complexas, onde geralmente os sistemas reais são compostos por um grande número de veículos, juntamente ao fato de que as solicitações por serviços ocorrem de forma temporária e espacialmente (Takeda, 2000). Lamosa et al. (2008) apontam que a abordagem de tratar o problema integrado pode ser utilizada para tratar sistemas complexos, como por exemplo serviços emergenciais, de aviação, questões militares e outros.

Os Location-Routing Problems (LRPs) têm como objetivo, dado um conjunto de clientes e possíveis locais de instalação de facilidades, determinar os locais onde as facilidades devem ser instaladas e designar as rotas destes locais aos clientes, objetivando a minimização do custo total, que inclui o custo de distribuição e de localização (Barreto et al., 2007). Algumas aplicações envolvendo problemas integrados na logística humanitária foram desenvolvidas, destacando-se as seguintes: Song e Zhang (2009) apresentam uma técnica de otimização para desenvolver um plano de evacuação para os moradores dependentes de trânsito em caso de catástrofes naturais, e onde foi utilizado o modelo LRP para localização de abrigos e 
roteirização para a minimização do tempo total de desocupação da área afetada; Yi e Özdamar (2007) propõem um problema de localização e roteirização para atender à demanda onde o atendimento é realizado por profissionais de saúde (médicos), assim como na locomoção das vítimas; Campbell et al. (2008) apresentam duas funções objetivos diferentes para o roteamento de veículos que transportam suprimentos para locais afetados por desastres; Lin et al. (2012) sugeriram a localização de armazéns temporários em torno de áreas afetadas por terremotos, com veículos e os recursos necessários para melhorar os processos logísticos de urgência a desastres; Ukkusuri e Yushimito (2008) apresentam uma abordagem de problemas conjunto de localização e roteirização, utilizando programação inteira, com intuito na preparação para o atendimento à população atingida por desastres de início rápido, causando rupturas na rede de transporte.

\section{Situações de seca e enchente no Estado do Amazonas}

Em 2005 e 2010 foram registradas as maiores secas onde o nível dos rios e lagos baixou de forma jamais vista pelas comunidades do interior do Estado do Amazonas. Segundo Souza e Almeida (2010), houve uma grande mortandade de peixes, prejuízos tanto quanto para a produção agrícola da várzea como para a criação de animais, e onde o rio Amazonas ficou com o nível muito baixo, prejudicando a navegação no Alto Solimões. Em 2009 e 2012 foram registradas as maiores cheias onde o nível dos rios subiu consideravelmente atingindo diversas famílias da região. As casas dos ribeirinhos (moradores da várzea) foram invadidas pela água do rio ocorrendo assim a migração para a cidade ou para terras-firmes, no entanto, a maioria optou em permanecer em suas residências, construindo um novo assoalho (conhecido como maromba).

Esses moradores enfrentam frequentemente a escassez de recursos na ocorrência de cheias e secas anormais, impossibilitando uma vida digna e resultando na realocação dos mesmos seja através da migração voluntária ou forçada. A ausência de políticas voltadas para assistir os moradores da várzea acaba forçando diversas famílias a migrarem para a cidade, na expectativa de serem assistidas (ALENCAR, 2005). Nogueira e Mainbourg (2010) afirmam que o êxodo rural proporcionou o empobrecimento econômico do campo. Além de promover a perda dos conhecimentos tradicionais para as futuras gerações na região (SILVA, 2008). A maioria da população que ainda continua habitando no interior do Estado está ligada ao cultivo agrícola e por possuírem uma "ligação" ou "identidade" com o lugar.

Como visto, as enchentes e especialmente as secas têm se apresentado com uma frequência e uma intensidade cada vez maiores, com secas extremas causando importantes prejuízos econômicos, sociais e ecológicos em consideráveis áreas das Américas do Norte e do Sul, Austrália, Europa e Ásia, e enchentes afetando a vida de milhões de pessoas no Sudeste Asiático e nas Américas.

Isso não é diferente no Estado do Amazonas, onde tem sido presenciadas grandes secas e cheias nos últimos anos. A ocorrência contínua destes eventos extremos mostrou a vulnerabilidade da população e dos órgãos competentes na assistência humanitária, expondo vítimas a situações vexatórias. A vazante/seca é um dos pontos de fragilização da logística e planejamento dos transportes da região. Desta maneira, em razão da dinâmica regional, é preciso haver esforços com mecanismos logísticos de proteção contra tais períodos de isolamento (KUWAHARA et al. 2012). Para tanto, providências podem e devem ser tomadas, pois observações indicam a tendência de que efeitos advindos das condições de sazonalidade na região acentuem-se nos próximos anos.

\section{Descrição da proposta}

O território do Estado do Amazonas possui características geográficas e geológicas distintas das demais regiões brasileiras; é uma conjuntura ambiental-social única no mundo inteiro. Diferente de outras catástrofes não anunciadas, e de difícil previsão, no Estado do Amazonas as secas e enchentes se repetem de forma sazonal. 0 desenvolvimento e a sobrevivência das comunidades que vivem no interior deste Estado dependem totalmente do transporte hidroviário, pois quase todo abastecimento básico é feito através de suas vias, as quais são usadas também para transportar pessoas que vivem nessas localidades. Esses problemas logísticos e de acessibilidade que dificultam o desenvolvimento e a promoção da qualidade de vida aos moradores da região. Atualmente no Amazonas o modal primário utilizado para assistência humanitária é modal aéreo - decorrente da falta de planejamento, da cultura da "necessidade de última hora" existente, das condições adversas dos rios e das grandes distâncias entre os municípios a serem atendidos.

A proposta deste modelo visa a construção de uma relação entre os conhecimentos já fundamentados pela literatura relacionada à Logística Humanitária no que diz respeito a preparação de eventos naturais prejudiciais ao homem, adequando-o a estruturas e meios de transporte, formando um escopo de planejamento de instalações humanitárias. Para tanto, e baseando-se no modelo de planejamento de instalações proposto por Lee (1998), foi elaborado etapas para localizações de Centrais de Abastecimento e Treinamento Humanitário (CATHs) e distribuição de suprimentos, conforme Figura 1.

A Figura 1 apresenta, resumidamente, o modelo conceitual para localização de instalações humanitárias e distribuição de suprimentos proposto por esta pesquisa. Fundamentalmente, as etapas do modelo devem seguir as seguintes atividades: i) determinar o local da instalação humanitária considerando municípios em área de risco afetados pelos desastres naturais/fenômenos naturais, no âmbito regional; ii) realizar um planejamento do tamanho das instalações de acordo com a demanda, em nível local; iii) elaborar um planejamento para determinar os tipos de suprimento para cada tipo de desastre ocorrido nos períodos estabelecidos, também em nível local; iv) elaborar um plano de rotas para entrega de suprimentos para as instalações, no âmbito regional. 0 cronograma das etapas e atuação do modelo informa cada etapa em função do tempo para localização e seleção das localidades, planejamento do tamanho das instalações, planejamento dos tipos de suprimentos entregues e finalmente, planejamento de rotas para entrega de suprimentos. Este trabalho tem como objetivo propor um modelo que facilite a locação de instalações humanitárias, armazenagem e distribuição de suprimentos, utilizando o transporte fluvial como modal primário, característica principal da região objeto de estudo. 


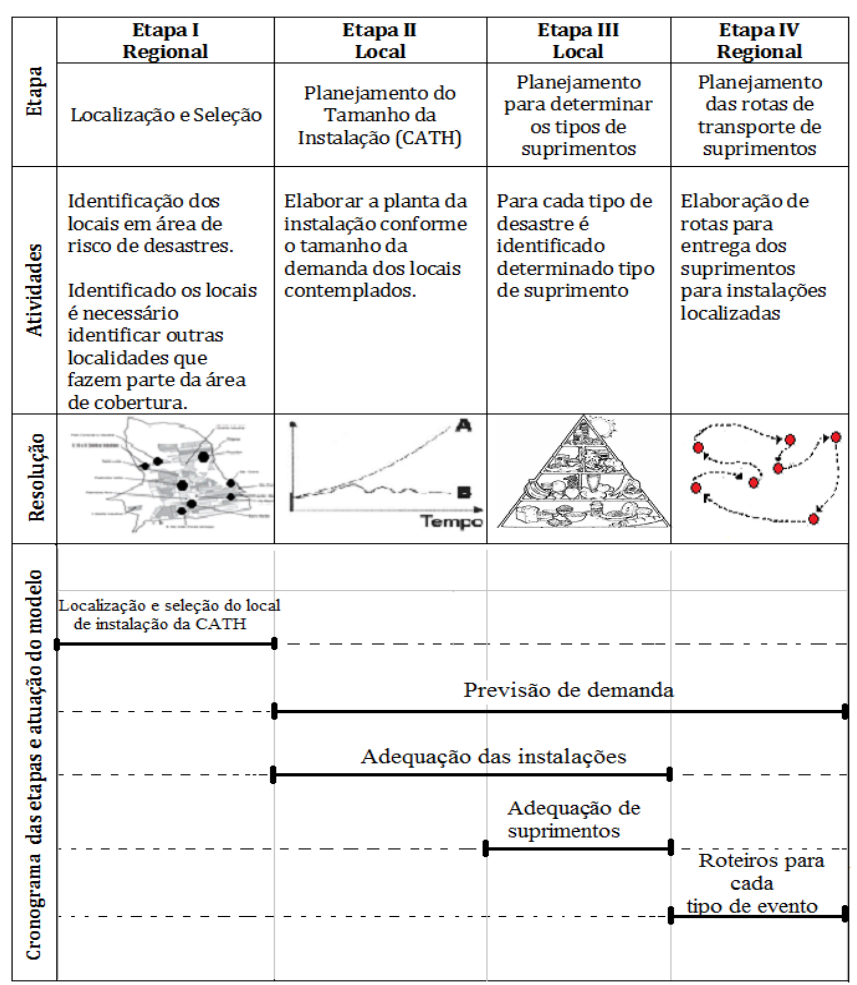

Figura 1: Modelo conceitual proposto para localização de instalações humanitárias e distribuição de suprimentos.

A Etapa I tem como objetivo a missão da Logística Humanitária onde suas instalações devem se localizar. Para que o resultado desta etapa seja coerente é necessário que as determinadas atividades sejam cumpridas. A primeira é identificar os locais em área de risco de desastres, conforme o modelo de planejamento de instalações elaborado por Lee (1998). A segunda é "identificar localidades que fazem parte da área de cobertura", que relaciona-se com os requisitos do Problema de Localização de Máxima Cobertura (Maximal Covering Location Problem - PLMC), que tem como objetivo localizar um número pré-estabelecido de facilidades (m facilidades), compatível com os recursos disponíveis, tal que a máxima população possível de uma dada região seja coberta a menos de uma distância crítica S predefinida (GALVÃo et al., 2003). A Etapa II ocorre o planejamento do tamanho da instalação, onde é necessário estabelecer as dimensões da instalação de acordo com o tamanho da demanda dos locais contemplados. Nessas instalações é previsto o recebimento de kits de higiene pessoal, alimentação, medicamentos, kits para os hospitais e purificadores de água, onde a população poderá sofrer com a escassez de água potável águas, entre outros, além de treinamento para população local. Na Etapa III, ocorre o planejamento para determinar os tipos de suprimentos, é importante identificar quais itens são fundamentais para sobrevivência das pessoas para cada tipo de desastre, realizando em determinados períodos a entrega dos suprimentos. A Etapa IV, é realizada a elaboração de rotas para entrega de suprimentos para instalações localizadas, que relaciona-se com Problema do Caixeiro Viajante ("Traveling Salesman Problem"), que tem como meta encontrar um roteiro de menor distância ou custo que percorre um conjunto de cidades, sendo que as mesmas deverão ser visitadas exatamente uma vez (Goldbarg e Luna 2000).

\section{Conclusão}

O trabalho buscou apresentar contribuições que abrangem a pesquisa operacional, mostrando a importância na solução de localização de instalações e distribuição de serviços, no campo da logística humanitária.

0 principal resultado foi ter identificado métodos de solução em problemas reais que envolvem modelos matemáticos integrados. As contribuições encontradas têm como objetivo em melhorar a capacidade de resposta, como minimizar o tempo de resposta, à distância e o custo de transporte, com intuito em resolver problemas em lugares, regiões ou países onde existe um histórico de desastres. Dessa maneira, dada a característica intrínseca aos problemas de localização e de roteirização, os modelos integrados que considerem esses dois aspectos passam a apresentar uma maior aplicação no processo de tomada de decisão em problemas complexos reais nos quais ambas as áreas precisam ser consideradas.

A construção de instalações humanitárias em municípios no interior do Estado do Amazonas que utilizem o transporte fluvial nas calhas o rios busca obter resultados como menor tempo de resposta a situações de perigo e diminuir o impacto destes eventos no dia-a-dia das populações que ali vivem e sofrem das consequências destes eventos associados à sazonalidade e as cheias e secas dos rios do Estado do Amazonas. Os desafios logísticos presentes nesse Estado se dão graças a grande dispersão geográfica dos municípios e da dificuldade de acesso a muitas regiões, justificando a busca por métodos eficientes para reduzir os custos de preparação e a migração (êxodo rural) para a capital. Tais ações podem fazer com que a população se fixe no campo fortalecendo a agricultura familiar e principalmente a cultura ribeirinha.

A forma de como é realizada a entrega de suprimentos atualmente, na "necessidade de última hora", gera um custo grande aos cofres públicos, pois se faz uso em demasia do modal aéreo e ocorre quando a situação já beira a criticidade, havendo o "sufocamento" da população necessitada dada a demora das autoridades em autorizar a ajuda financeira - não prevista - para a realização e a entrega de suprimentos básicos que poderiam já estar mais próximos destas comunidades.

Desse modo, este estudo propõe a utilização de um sistema de transporte hidroviário eficiente, que considere a utilização de instalações próximas a cidades de referência e que possam suprir as necessidades urgentes das populações afetadas. 0 enfrentamento destas adversidades de forma planejada, embasado não pelo empirismo de órgãos competentes, mas com a ajuda de modelos que considerem as características dos transportes da região, pode tornar-se um marco importante para a 
sobrevivência destas comunidades, em especial àquelas do interior do Estado do Amazonas, as quais são, em geral, as mais afetadas e as mais negligenciadas pelas autoridades.

Sugerem-se como pesquisas futuras acerca do tema, abordagens de modelos matemáticos que considerem tempos de viagem, instalações em diferentes localidades, auxílio da logística militar, mapeamento de zonas de perigo, etc. Além disso, é de interesse também realizar experimentos de dimensionamento da frota e desenvolver políticas de estoques para responder aos desastres de início lento, que contribuem para as decisões de localização de facilidades.

\section{Referências}

Alencar, E. F. (2005). Políticas Públicas e (in) Sustentabilidade Social: O caso de comunidades de várzea no Alto Solimões, Amazonas. In Lima, D. (Org.) Diversidade Socioambiental nas várzeas dos rios Amazonas e Solimões - perspectivas para o desenvolvimento da sustentabilidade. Ibama, Pró Várzea, Manaus, p. 59-99.

Balcik, B., Beamon, B. M., Krejci, C. C., Muramatsu, K. M. \& Ramirez, M. (2010). Coordination in humanitarian relief chains: Practices, challenges and opportunities. International Journal of Production Economics, 1(126), p. 22-34.

Balcik, B. \& Beamon, B.M. (2008). Facility Location in humanitarian relief. International Journal of Logistics: Research and Applications. 11(2), p. 101-121.

Barreto, S., Paixão, C. F. J. \& Santos, B. S. (2007). Using clustering analysis in a capacitated location-routing problem, European Journal of Operational Research, (179), p. 968-977.

Campbell, A.M., Vandenbussche, D. \& Hermann, W. (2008). Routing for relief efforts. Transportation Science 42 (2):127-145, 2008.

Cunha, C. B. (2000). Aspectos Práticos da Aplicação de Modelos de Roteirização de Veículos a Problemas Reais. Transportes, RJ, 8(2), p. 5174.

Galvão, R. D., Chiyoshi, F. Y.; Espejo, L. G. A., Rivas, M. P. A. (2003). Solução do problema de localização de máxima disponibilidade utilizando o modelo hipercubo. Pesquisa Operacional, SOBRAPO, v. 23, n.1, p. 61-78.

Goldbarg, M. C. \& Luna, H. P. L. (2000). Otimização combinatória e programação linear: modelos e algoritmos. Rio de Janeiro: Editora Campos.

Kovács, G. \& Spens K. (2007). Humanitarian logistics in disaster relief operations. International Journal of Physical Distribution e Logistics Management, 37(2), p. 99-114.

Kuwahara, N., Neto, J. C. L. \& Abensur, T. C. (2012). Modelagem de previsão de navegabilidade em rios da Amazônia: ferramenta web de suporte aos usuários do transporte aquaviário. Journal of Transport Literature, 6(3), p. 60-89.

Lamosa, M. J. P., De Marchi, M. M. \& Santos, C. L. R. (2008). O Problema de localização de máxima cobertura integrado ao problema de roteamento. In: XL Simpósio Brasileiro de Pesquisa Operacional, João Pessoa. XL SBPO. Rio de Janeiro: SOBRAPO, 2008. p. 1721 1729.

Lee, Q. (1998). Projeto de instalações e do Local de Trabalho. 1. Ed. São Paulo: IMAM,

Lin, Y-H, Battar, Rogerson P, Blatt A, \& Flanigan M. (2012). Locating of temporary depots to facilitate relief operations after an earthquake. Socio-Economic Planning Sciences (46), p. 112-123.

Lobo, D. S. (2003). Dimensionamento e otimização locacional de unidades de educação infantil. 142 pág. Tese (Doutorado em Engenharia de Produção) Universidade Federal de Santa Catarina, Florianópolis.

Mclachlin, R. \& Larson, P. D. (2011). Building humanitarian supply chain relationschip: lessons from leading practitioners. Journal of Humanitarian Logistics and Supply Chain Management, 1(1), p. 32-49.

Nogueira, A. C. F. \& Mainbourg, E. M. T. (2010). A comunidade do pau rosa/Amazonas e a relação entre natureza, cultura e o processo saúde/doença. Saúde e Sociedade (USP. Impresso), (19), p. 22-34.

Nogueira, C.W., Gonçalves, M.B. \& Novaes A.G. (2008). A logística humanitária e medidas de desempenho: A perspectiva da cadeia de assistência humanitária. Artigo. Anais do XXII Congresso de Pesquisa e Ensino em Transportes. Novembro. Fortaleza

Overstreet, R. E., Hall, D., Hanna, J. B. \& Rainer, JR. R. K. (2011). Research in humanitarian logistics. Journal of Humanitarian Logistics and Supply Chain Management, 1(2), p. 114-131.

Samed, M. M. A. \& Goncalves, M. B. (2013). O Estado Atual dos Problemas de Localização e suas Contribuições para as Pesquisas da Logística Humanitária no Brasil. In: XXVII ANPET, 2013, Belém, PA. Anais do XXVII ANPET.

Silva, A. L. (2008). Animais medicinais: conhecimento e uso entre as populações ribeirinhas do rio negro, Amazonas, Brasil. Boletim do Museu Paraense Emílio Goeldi. Ciências Humanas, 3, p. 343-357.

Song, R., S. He \& L. Zhang (2009). Optimum transit operations during the emergency evacuations. Journal of Transportation Systems Engineering and Information Technology, 9(6), p. 154-160.

Souza, J. C. R. de \& Almeida, R. A. de, (2010). Vazante e enchente na Amazônia brasileira: impactos ambientais, sociais e econômicos. In: VI Seminário Latino Americano de Geografia Física, 2010, Coimbra-Portugal. Sustentabilidade da "Gaia": ambiente, ordenamento e desenvolvimento.

Takeda, R. A. (2000). Uma contribuição para avaliar o desempenho de sistemas de transporte emergencial de saúde. Tese (doutorado) Escola de Engenharia de São Carlos - Universidade de São Paulo.

Thomas, A. (2004). Elevating Humanitarian Logistics. International Aid e Trade Review.

Thomas, A. \& Kopezak, L. R. (2007). "Life-saving supply chains: Challenges and path forward," in Building Supply Chain Excellence in Emerging Economies, (H. L. Lee and C. Y. Lee, eds.), New York: Springer Science, p. 93-111.

Tomasini, R. \& Van Wassenhove, L. N. (2009). Humanitarian logistics. Insead Business Press, London, England.

Ukkusuri, S. \& Yushimito, W. (2008). A Location-Routing Approach for the Humanitarian PrePositioning Problem. Journal of Transportation Research Record, p. 18-25.

Yi, W. \& Özdamar, L. A. (2007). dynamic logistics coordination model for evacuation and support in disaster response activities. European Journal of Operational Research 179, p. 1177-1193.

\section{Abstract}

The activities that are encompassed by the Humanitarian Logistics can be realized pre or post-disaster, more importantly in the Preparation and Response phases in activities such as locating facilities and routing. In the Amazon State in Brazil, due to the anomalies of the pre-announced natural phenomenon's, supplying issues affect the survival of the local population, mostly in the riverside communities. For such, the main goal of this paper is to present a reference model for locating facilities and routing the supply distribution for humanitarian facilities as an alternative for immediate response for those affected by these natural seasonal catastrophe events that occur in the Amazon State. 